\title{
Hyperoctahedral Eulerian Idempotents, Hodge Decompositions, and Signed Graph Coloring Complexes
}

\author{
Benjamin Braun* \\ Department of Mathematics \\ University of Kentucky \\ Lexington, KY 40506 U.S.A. \\ benjamin . braun@uky . edu
}

\author{
Sarah Crown Rundell \\ Department of Mathematics and Computer Science \\ Denison University \\ Granville, OH, 43023 U.S.A. \\ rundells@denison.edu
}

Submitted: Aug 7, 2013; Accepted: May 7, 2014; Published: May 22, 2014

Mathematics Subject Classifications: 05C15; 18G35; 05C31

\begin{abstract}
Phil Hanlon proved that the coefficients of the chromatic polynomial of a graph $G$ are equal (up to sign) to the dimensions of the summands in a Hodge-type decomposition of the top homology of the coloring complex for $G$. We prove a type B analogue of this result for chromatic polynomials of signed graphs using hyperoctahedral Eulerian idempotents.
\end{abstract}

Keywords: Chromatic polynomial; signed graph; Hodge decomposition; Eulerian idempotent; coloring complex

\section{Introduction}

Let $G$ denote a finite graph and $\chi_{G}(\lambda)$ its chromatic polynomial. The coloring complex $\Delta_{G}$ was defined by Einar Steingrímsson [18] in order to provide a Hilbert-polynomial interpretation of $\chi_{G}(\lambda)$. While Steingrímsson's original definition of $\Delta_{G}$ was motivated by algebraic considerations, the coloring complex can also be obtained as the link complex for a hyperplane arrangement, using techniques developed by Jürgen Herzog, Vic Reiner, and Volkmar Welker [11]. Coloring complexes have many interesting properties. Jakob Jonsson proved [13] that $\Delta_{G}$ is homotopy equivalent to a wedge of spheres in fixed dimension, with the number of spheres being one less than the number of acyclic orientations of $G$. Axel Hultman [12] proved that $\Delta_{G}$, and in general any link complex for a

*Partially supported by the National Security Agency through award H98230-13-1-0240. 
sub-arrangement of the type A or type B Coxeter arrangement, is shellable. Further, $\Delta_{G}$ admits a convex ear decomposition, as shown by Patricia Hersh and Ed Swartz [10].

A fascinating result due to Phil Hanlon [8] is that (up to sign) the $j$-th coefficient of $\chi_{G}(\lambda)$ is equal to the dimension of the $j$-th summand in a Hodge-type decomposition of the top homology of $\Delta_{G}$. Hanlon's Hodge decomposition is obtained using the Eulerian idempotents in the group algebra of the symmetric group $S_{n}$. These are the elements $e_{n}^{(j)} \in \mathbb{C}\left[S_{n}\right]$ defined by

$$
e_{n}(x)=\sum_{j=1}^{n} x^{j} e_{n}^{(j)}=\sum_{\pi \in S_{n}}\left(\begin{array}{c}
x+n-\operatorname{des}(\pi)-1 \\
n
\end{array}\right) \operatorname{sgn}(\pi) \pi .
$$

These first arose in work of various authors in the 1980's. Murray Gerstenhaber and Samuel Schack [6] proved that all splitting sequences for Hochschild homology arise as linear combinations of Eulerian idempotents, which in certain cases coincides with Hodge decompositions for smooth compact complex varieties; similar work was independently introduced by Jean-Louis Loday [14]. A nice introduction to these results can be found in another paper due to Hanlon [7, Section 1]. The chain complex defining Hochschild homology is quite similar to the chain complex for $\Delta_{G}$, and thus Hanlon was able to adapt the Eulerian idempotent splitting techniques in Hochschild homology to produce a similar decomposition for the top homology of $\Delta_{G}$.

The coloring complex construction can be extended to hypergraphs, and Eulerian idempotents continue to play a role in this setting. Combinatorial and topological properties of hypergraph coloring complexes were investigated by Felix Breuer, Aaron Dall, and Martina Kubitzke [3], who found that many of the nicest properties of graph coloring complexes are lost in the transition to hypergraphs, e.g. Cohen-Macaulayness, partitionability, being a wedge of spheres, etc. Hypergraph coloring complexes were also considered by Jane Long and the second author [15]. They show that the homology of hypergraph coloring complexes admits a Hodge decomposition induced by Eulerian idempotents, and that the coefficients of the chromatic polynomial of a hypergraph are essentially the Euler characteristics of the Hodge subcomplexes, up to sign. The second author [17] investigated the special case of $k$-uniform hypergraphs, showing that their coloring complexes are shellable and that their cyclic coloring complexes have a certain homology group whose dimension is given by a binomial coefficient.

The Eulerian idempotents play key roles in other contexts as well. Adriano Garsia [5] and Christophe Reutenauer [16] studied Eulerian idempotents in their work on free Lie algebras. Persi Diaconis and Jason Fulman [4] show that the Eulerian idempotents are (up to the sign involution) eigenvectors of an "amazing" matrix arising from the study of "carries" in addition algorithms. They also show that this matrix is related to the Veronese construction in commutative algebra. Phil Hanlon and Patricia Hersh [9] prove that the homology of the complex of injective words admits a Hodge decomposition, where the dimension of the $k$-th Hodge summand is equal to the number of derangements with exactly $k$ cycles. These and other results are all-the-more fascinating due to their type B extensions. The type B Eulerian idempotents, defined in Section 4, were originally defined by François Bergeron and Nantel Bergeron [2]. They proved type B extensions 
of several of the type A results given above. The Eulerian idempotents in types A and $\mathrm{B}$ also play an interesting role in shuffling problems, as discussed in several of the papers just referenced.

Given the variety of interesting applications of Eulerian idempotents, we believe that a type B version of Hanlon's result regarding $\chi_{G}(\lambda)$ is of interest. The goal of this paper is to prove Theorem 26, which provides the desired extension in the setting of signed graph chromatic polynomials. Section 2 contains a review of basic properties of signed graphs and their chromatic polynomials. Section 3 discusses signed graph coloring complexes and hyperoctahedral group actions on them. In Section 4, we prove that the type B Eulerian idempotents induce a Hodge-type decomposition on the top homology of each signed graph coloring complex. In Section 5 we prove our main result.

\section{Signed graphs and chromatic polynomials}

This section is based on Zaslavsky's papers [19, 20].

Definition 1. A signed graph $G$ on the vertex set $[n]$ is a multiset $E$ of one-element subsets of $[n]$, called half-edges, and two-element subsets of $[n]$, called edges, together with a sign map $\sigma: E \cap\left(\begin{array}{c}{[n]} \\ 2\end{array}\right) \rightarrow\{1,-1\}$ such that $\sigma^{-1}(1)$ and $\sigma^{-1}(-1)$ are each the edge set of a simple graph on [n]. For an edge $e \in E$, if $\sigma(e)=1$, then $e$ is called a positive edge of $G$. If $\sigma(e)=-1$, then $e$ is called a negative edge of $G$.

Example 2. Let $G$ have vertex set $\{1,2,3\}$, positive edge $\{1,2\}$, negative edges $\{1,2\}$ and $\{2,3\}$, and half-edge $\{1\}$. We schematically represent $G$ using solid half-lines and lines for half-edges and positive edges, respectively, and using dotted lines for negative edges, as demonstrated in Figure 1.

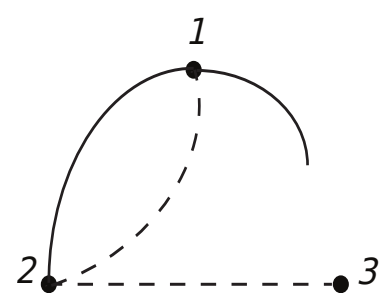

Figure 1:

Definition 3. Let $G$ be a signed graph on $[n]$. A c-coloring of $G$ is a map

$$
\phi:[n] \rightarrow\{-c,-c+1, \ldots,-1,0,1, \ldots, c\} .
$$

A $c$-coloring $\phi$ is proper if $\phi(i) \neq \phi(j)$ for all positive edges $\{i, j\}$ in $G, \phi(i) \neq-\phi(j)$ for all negative edges $\{i, j\}$ in $G$, and $\phi(i) \neq 0$ for all half-edges $i \in E(G)$. Denote by $\chi_{G}(2 c+1)$ the number of proper $c$-colorings of $G$. 
Theorem 4 (Zaslavsky [20]). For $G$ a signed graph on $[n]$, the function $\chi_{G}(2 c+1)$ is given by a polynomial of degree $n$.

Example 5. For $G$ as in Example 2, we have

$$
\chi_{G}(\lambda)=(\lambda-1)(\lambda-2)(\lambda-1)=\lambda^{3}-4 \lambda^{2}+5 \lambda-2,
$$

where $\lambda$ is the number of colors in a set of colors containing the color 0 . Note that evaluating $\chi_{G}(\lambda)$ at $\lambda=2 c+1$ yields the number of proper $c$-colorings of $G$.

The key to proving polynomiality of $\chi_{G}(2 c+1)$ is the relation between signed graphs, contractions, and deletions, which we will need subsequently. Contraction/deletion for signed graphs relies upon the idea of switching a signed graph at a vertex.

Definition 6. Let $G$ be a signed graph with sign map $\sigma$, and let $v$ be a vertex of $G$. We say that the signed graph $G^{\prime}$ is obtained by switching $G$ at $v$ if the vertex and edge sets for $G^{\prime}$ are $V(G)$ and $E(G)$, while the sign map $\sigma^{\prime}$ for $G^{\prime}$ is given by

$$
\sigma^{\prime}(\{i, j\}):=\left\{\begin{array}{ll}
-\sigma(\{i, j\}) & \text { if } v=i \text { or } v=j \\
\sigma(\{i, j\}) & \text { else }
\end{array} .\right.
$$

If $H$ is obtained from $G$ by a finite sequence of switches, we say that $G$ and $H$ are switching equivalent.

Thus, one switches from $G$ to $G^{\prime}$ at $v$ by negating the sign on all edges in $G$ incident with $v$. The following proposition, demonstrating the role played by switching, is simple to prove.

Proposition 7. If $G$ and $H$ are switching equivalent, then

$$
\chi_{H}(2 c+1)=\chi_{G}(2 c+1) .
$$

Definition 8. Let $G$ be a signed graph with an edge $e=\{i, j\} \in E(G)$ with sign $\sigma(e)$. The deletion of $G$ by $e$, denoted $G \backslash e$, is the signed graph obtained by removing $e$ from $E(G)$. A contraction of $G$ by $e$, denoted $G / e$, is a signed graph in the same switching class as the graph obtained from $G$ by the following process (which is well-defined up to switching equivalence).

- If $\sigma(e)=1$, then delete $e$ from $E(G)$ and contract as in the ordinary graph case by identifying $i$ and $j$ in $V(G)$. When $\{i, j\}$ is also present in $E(G)$ as a negative edge, add a half-edge at the vertex given by $i=j$ after contracting (if this half-edge is not already present).

- If $\sigma(e)=-1$, then first switch at an endpoint of $e$ so that $\sigma(e)=1$ and proceed as in the positive edge contraction case.

Given a half-edge $i \in E(G)$, the deletion of $G$ by $i$, denoted $G \backslash i$, is the signed graph obtained by removing $i$ from $E(G)$. The contraction of $G$ by $i$, denoted $G / i$, is the signed graph with vertex set $V(G) \backslash\{i\}$ and edge set $\{e \backslash\{i\} \mid e \in E(G)\}$. 
Note that $E(G)$ is a multiset, thus it is possible that two copies of $\{i, j\}$ are contained in $E(G)$ with different signs. If this is the case, then only one copy of $\{j\}$ is retained in the edge set of the deletion and contraction. The key property of contraction/deletion, and what makes it relevant for the proof of Theorem 4, is given next.

Proposition 9. Given a signed graph $G$ with positive edge e,

$$
\chi_{G}(2 c+1)=\chi_{G \backslash e}(2 c+1)-\chi_{G / e}(2 c+1) .
$$

A final fact we need is that when the chromatic polynomial is expressed as

$$
\chi_{G}(\lambda)=\sum_{j=1}^{n-1}(-1)^{n-j} c_{j}(G) \lambda^{j}+\lambda^{n},
$$

the $c_{j}$ 's are non-negative integers. This can be seen in several ways, e.g. by recognizing $\chi_{G}(\lambda)$ as the characteristic polynomial of the arrangement $\mathcal{B}_{G}$ defined in the next section.

\section{Signed graphic arrangements, coloring complexes, and group actions}

Our construction of signed graph coloring complexes involves the following hyperplane arrangement.

Definition 10. The type $B$ braid arrangement is the collection of hyperplanes

$$
\mathcal{B}_{n}:=\left\{H_{i j}^{+1} \mid 1 \leqslant i<j \leqslant n\right\} \cup\left\{H_{i j}^{-1} \mid 1 \leqslant i<j \leqslant n\right\} \cup\left\{H_{i} \mid 1 \leqslant i \leqslant n\right\}
$$

where $H_{i j}^{+1}=\left\{\left(x_{1}, \ldots, x_{n}\right) \in \mathbb{R}^{n} \mid x_{i}=x_{j}\right\}, H_{i j}^{-1}=\left\{\left(x_{1}, \ldots, x_{n}\right) \in \mathbb{R}^{n} \mid x_{i}=-x_{j}\right\}$, and $H_{i}=\left\{\left(x_{1}, \ldots, x_{n}\right) \mid x_{i}=0\right\}$.

The arrangement $\mathcal{B}_{n}$ induces a regular cell decomposition $\Delta_{\mathcal{B}_{n}}$ of the sphere $S^{n-1}$, which we describe using the choice of $\partial[-1,1]^{n}$ as our preferred representation of $S^{n-1}$. $\mathcal{B}_{n}$ induces a triangulation of $\partial[-1,1]^{n}$ where each vertex $v=\left(v_{1}, \ldots, v_{n}\right)$ of the resulting triangulation can be identified with a nonempty subset of $[n] \cup-[n]$ by the rule that for each $v_{i}, \pm i$ is included in the subset if $v_{i}= \pm 1$, respectively, and $i$ is not included in the subset if $v_{i}=0$. The faces of the triangulation are given by collections of vertices corresponding to chains (with respect to inclusion) of subsets of this type. Alternatively, given such a chain

$$
C:=Q_{1} \subset Q_{2} \subset Q_{3} \subset \cdots \subset Q_{r},
$$

we associate to $C$ the ordered set partition of $[n] \cup-[n]$ given by

$$
Q_{1}\left|Q_{2} \backslash Q_{1}\right| Q_{3} \backslash Q_{2}|\cdots| Q_{r} \backslash Q_{r-1} \mid[n] \cup[-n] \backslash Q_{r}
$$

It is clear that $C$ may be fully recovered from its associated partition. 
Example 11. The triangulation of $\partial[-1,1]^{3}$ induced by $\mathcal{B}_{3}$ is shown in Figure 2. The chamber marked $\mathrm{G}$ has vertices $(0,1,0),(0,1,-1)$, and $(-1,1,-1)$; thus, $\mathrm{G}$ is identified with the chain

$$
\{2\} \subset\{2,-3\} \subset\{-1,2,-3\}
$$

with associated partition $(2|-3|-1 \mid 1,-2,3)$. Similarly, the chamber marked B has vertices $(1,0,0),(1,-1,0)$, and $(1,-1,1)$, hence corresponds to the chain

$$
\{1\} \subset\{1,-2\} \subset\{1,-2,3\}
$$

with associated partition $(1|-2| 3 \mid-1,2,-3)$.

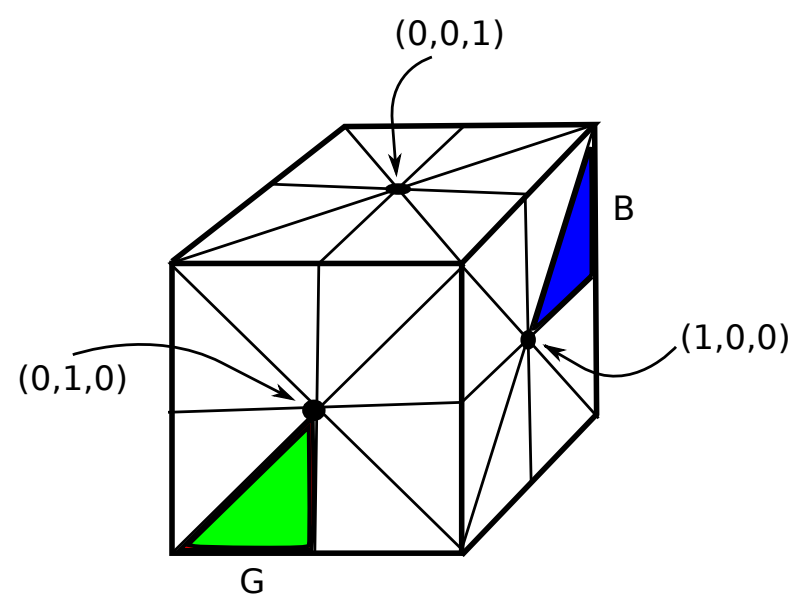

Figure 2:

Definition 12. Given a signed graph $G$ with sign map $\sigma$, the (signed) graphic arrangement corresponding to $G$ is the subarrangement $\mathcal{B}_{G}$ of $\mathcal{B}_{n}$ defined by

$$
\mathcal{B}_{G}=\left\{H_{i j}^{\sigma(\{i, j\})} \mid\{i, j\} \in E(G)\right\} \cup\left\{H_{i}:\{i\} \in E(G)\right\} .
$$

Example 13. Continuing with the signed graph of Example 2, we see that

$$
\mathcal{B}_{G}=\left\{\left(x_{1}, x_{2}, x_{3}\right) \in \mathbb{R}^{3} \mid \text { either } x_{1}=0, x_{1}=x_{2}, x_{1}=-x_{2} \text { or } x_{2}=-x_{3}\right\} .
$$

Before discussing the geometric manifestation of coloring complexes using $\mathcal{B}_{G}$, we will first define the coloring complex of a signed graph in a purely combinatorial manner, using the viewpoint of ordered set partitions developed above. For $G$ a signed graph on $[n]$, we say a subset $A \subset[n] \cup-[n]$ contains an edge of $G$ if one of the following two cases hold for some pair $\{a, b\} \subset A$.

- $\{a, b\}$ is a positive edge in $G$, or

- $a \in[n]$ and $b \in-[n]$, and $\{a,-b\}$ is a negative edge in $G$. 
Definition 14. Given a signed graph $G$, the coloring complex $\Delta_{G}$ is the simplicial complex whose facets are ordered set partitions $P_{1}\left|P_{2}\right| \cdots \mid P_{n}$ of $[n] \cup-[n]$ such that

1. $P_{n}=([n] \cup-[n]) \backslash \cup_{j=1}^{n-1} P_{j}$,

2. for each pair $\{j,-j\}$ with $j \in[n]$, either $j$ or $-j$ is contained in $P_{n}$, and

3. either there exists a unique non-singleton block $P_{i}$ with $1 \leqslant i \leqslant n-1$ that contains an edge of $G$, or

4. for some half-edge $i \in E(G),\{i,-i\} \subset P_{n}$.

The faces of $\Delta_{G}$ are formed by merging adjacent blocks in the partitions defining the facets. Thus, the vertices of $\Delta_{G}$ are given by partitions $P_{1} \mid([n] \cup-[n]) \backslash P_{1}$ where $P_{1}$ is obtained by merging an initial segment of blocks in one of the facets of $\Delta_{G}$ described above. The $r$-dimensional faces of $\Delta_{G}$ are the ordered set partitions with $r+2$ blocks. Note that the role of the empty set is taken by the trivial partition $[n] \cup-[n]$. As in the case for $\Delta_{\mathcal{B}_{n}}$, each partition $P_{1}\left|P_{2}\right| \cdots \mid P_{n}$ corresponds uniquely to a chain in $2^{[n] \cup-[n]}$ of the form

$$
\emptyset \subset P_{1} \subset P_{1} \cup P_{2} \subset \cdots \subset \cup_{i=1}^{j} P_{i} \subset \cdots \subset[n] \cup-[n] .
$$

Geometrically, the coloring complex arises as $\Delta_{G}=\mathcal{B}_{G} \cap \partial[-1,1]^{n}$. The space $\Delta_{G}$ inherits the simplicial triangulation described above via the restriction of $\Delta_{\mathcal{B}_{n}}$ to $\Delta_{G}$. The connection to the triangulation of $\partial[-1,1]^{n}$ induced by $\mathcal{B}_{n}$ is immediate from our previous discussion. We will freely use the notation $\Delta_{G}$ to denote both the topological space $\mathcal{B}_{G} \cap \partial[-1,1]^{n}$ and the abstract simplicial complex obtained after intersecting with $\Delta_{\mathcal{B}_{n}}$. Given this geometric observation, it follows that $\Delta_{G}$ is an example of a link complex of a subspace arrangement, resulting in the following theorem.

Theorem 15. (Hultman, [12, Theorem 4.2]) For any signed graph $G, \Delta_{G}$ is shellable, hence is homotopy equivalent to a wedge of spheres of dimension $n-2$.

One reason the complex $\Delta_{G}$ is important is that it provides a path through which we can interpret chromatic polynomials as Hilbert polynomials of graded algebras.

Theorem 16. (Hultman, [12, Corollary 5.8]) Let $k\left[\operatorname{cone}\left(\Delta_{G}\right)\right]$ be the Stanley-Reisner ring of the cone over $\Delta_{G}$. The Hilbert polynomial of $k\left[\operatorname{cone}\left(\Delta_{G}\right)\right]$ is given by

$$
F\left(k\left[\operatorname{cone}\left(\Delta_{G}\right)\right] ; c\right)=(2 c+1)^{n}-\chi_{G}(2 c+1) .
$$

Example 17. Let $G$ be as in Example 2, hence $\mathcal{B}_{G}$ as in Example 13. The complex $\Delta_{G}$, arising as a subcomplex of the triangulation of $\partial[-1,1]^{3}$ shown in Figure 2, is given in Figure 3. It is straightforward to verify that $\Delta_{G} \simeq \vee_{i=1}^{11} S^{1}$.

We will later need to undertake a detailed analysis of the boundary operators for $\Delta_{G}$, for which the following notation is needed. Let $d_{i}$ denote the map on the $r$-faces of $\Delta_{G}$ defined by:

$$
d_{i}\left(P_{1}|\cdots| P_{i}\left|P_{i+1}\right| \cdots \mid P_{r+2}\right)=\left(P_{1}|\cdots| P_{i-1}\left|P_{i} \cup P_{i+1}\right| P_{i+2}|\cdots| P_{r+2}\right) .
$$




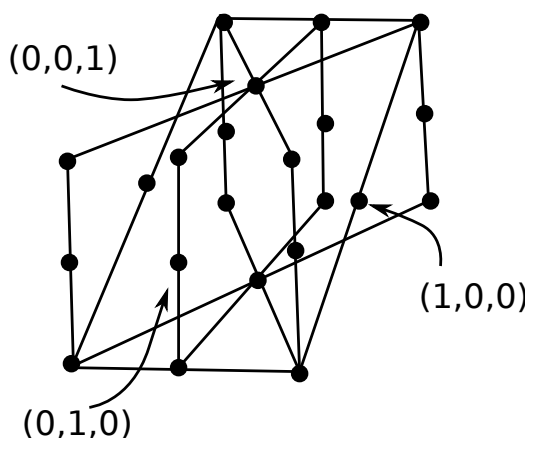

Figure 3:

The boundary operator on the $r$-chains of $\Delta_{G}$ is then defined by

$$
\partial_{r}=\sum_{i=1}^{r+1}(-1)^{i-1} d_{i} .
$$

Note that since $\Delta_{G}$ is homotopic to a wedge of spheres of dimension $n-2$, the reduced homology of $\Delta_{G}$ is non-vanishing only in dimension $n-2$.

Another reason that signed graph coloring complexes are interesting is that their chain spaces admit a family of actions by hyperoctahedral groups, which we will now introduce. All relevant background regarding hyperoctahedral groups can be found in $[2$, Section 8.1]. Let $B_{n}$ denote the $n$-th hyperoctahedral group, i.e., $B_{n}$ is the set of all permutations of $[-n, n]$ such that $\pi(-i)=-\pi(i)$ for all $i \in\{0,1, \ldots, n\}$. Note that $\pi(0):=0$ for all $\pi \in B_{n}$. For an element $\pi \in B_{n}$, we say the window for $\pi$ is

$$
\pi=[\pi(1) \pi(2) \cdots \pi(n)] ;
$$

thus, the window for $\pi$ is analogous to one-line notation for the symmetric group. Recall that for an element $\pi \in B_{n}$, the sign of $\pi$ is

$$
\operatorname{sgn}(\pi):=(-1)^{\ell(\pi)}
$$

where $\ell(\pi)$ is the length of $\pi$. Recall also that the descent statistic for $\pi \in B_{n}$ is

$$
\operatorname{des}(\pi):=\#\{i \in[0, \ldots, n-1] \mid \pi(i)>\pi(i+1)\} .
$$

Set $P_{-i}:=-P_{i}$. For any signed graph $G$, an element $\pi \in B_{r+1}$ acts on $C_{r}\left(\Delta_{G} ; \mathbb{C}\right)$, the space of $r$-chains of the coloring complex for $G$, by extending linearly the action on basis elements given by

$$
\pi\left(P_{1}\left|P_{2}\right| \cdots\left|P_{r+1}\right| P_{r+2}\right)=\left(P_{\pi^{-1}(1)}|\cdots| P_{\pi^{-1}(r+1)} \mid P_{r+2}^{\prime}\right),
$$

where $P_{r+2}^{\prime}$ is $P_{r+2}$ with $j \in P_{r+2}$ changed to $-j \in P_{r+2}^{\prime}$ if $\pi$ changed the sign on the block containing $j$. Informally, $\pi$ acts by permuting the first $r+1$ blocks in the partitions defining the $r$-faces of $\Delta_{G}$ by $\pi^{-1}$, changing the signs of all elements in blocks where a sign change occurs on the index, and subsequently modifying $P_{r+2}$ to account for those sign changes. 
Example 18. Let $\pi=\left[\begin{array}{ll}2-1-3\end{array}\right] \in B_{3}$, hence $\pi^{-1}=\left[\begin{array}{ll}-2 & 1-3\end{array}\right]$. For

$$
\tau:=(1,3|-2,5| 6 \mid-1,2,-3,4,-4,-5,-6) \in C_{2}\left(\Delta_{B_{6}}\right),
$$

we have that

$$
\pi(\tau)=(2,-5|1,3|-6 \mid-1,-2,-3,4,-4,5,6) .
$$

\section{Eulerian idempotents and type B Hodge decompo- sitions}

Our goal in this section is to prove Theorem 21, stating that $H_{n-2}\left(\Delta_{G}\right)$ admits a particular type of direct sum decomposition called a type $B$ Hodge decomposition. In order to state the theorem, we must begin with a discussion of the following family of idempotents defined by F. Bergeron and N. Bergeron [1].

Definition 19. The Eulerian idempotents of type B are elements $\tilde{\rho}_{n}^{(j)} \in \mathbb{C}\left[B_{n}\right]$ defined by

$$
\tilde{\rho}_{n}(x)=\sum_{j=0}^{n} x^{j} \tilde{\rho}_{n}^{(j)}:=\sum_{\pi \in B_{n}}\left[\frac{\prod_{k=1}^{n}(x-2 \operatorname{des}(\pi)+2 k-1)}{2^{n} n !}\right] \operatorname{sgn}(\pi) \pi .
$$

We will need two key properties of the type B Eulerian idempotents. First, Bergeron and Bergeron [1, Section 4] proved that

$$
\tilde{\rho}_{n}^{(r)} \tilde{\rho}_{n}^{(s)}=\delta_{r, s} \tilde{\rho}_{n}^{(r)}
$$

Second, evaluating (2) at $x=1$ yields

$$
\sum_{j=0}^{n} \tilde{\rho}_{n}^{(j)}=\mathrm{id}
$$

after observing that

$$
\frac{\prod_{k=1}^{n}(1-2 \operatorname{des}(\pi)+2 k-1)}{2^{n} n !}=\left(\begin{array}{cl}
-\operatorname{des}(\pi)+n \\
n
\end{array}\right)=\left\{\begin{array}{ll}
1 & \text { if } \operatorname{des}(\pi)=0 \\
0 & \text { else }
\end{array} .\right.
$$

Thus, the $\tilde{\rho}_{n}^{(j)}$ form a family of orthogonal idempotents in $\mathbb{C}\left[B_{n}\right]$ that sum to the identity element.

Remark 20. It is not clear from Definition 19 why these elements should be orthogonal idempotents, nor that they should have any other special properties. In the opinion of the authors, the Eulerian idempotents are rather mysterious in general, though they exhibit many remarkable properties as mentioned in Section 1. 
The Eulerian idempotents do admit the following concrete definition, which will be useful in this section. Set

$$
\tilde{l}_{n}^{(j)}:=(-1)^{j-1} \sum_{\substack{\pi \in B_{n} \\
\operatorname{des}(\pi)=j}} \operatorname{sgn}(\pi) \pi \quad \text { and } \quad \tilde{\lambda}_{n}^{(j)}=\sum_{i=0}^{j}(-1)^{i}\left(\begin{array}{c}
n+i \\
i
\end{array}\right) \tilde{l}_{n}^{(j-i)} .
$$

It is straightforward, though tedious, to verify that

$$
\tilde{\lambda}_{n}^{(j)}=(-1)^{j-1} \tilde{\rho}_{n}(2 j+1)
$$

by evaluating (2) at $2 j+1$. Due to this relation, it follows that the $\tilde{\lambda}_{r+1}^{(j)}$ can be used to define the $\tilde{\rho}_{r+1}^{(j)}$ via an invertible Vandermonde matrix.

We are now able to give our main result of this section, which states that the chain complex for the signed graph coloring complex admits a direct sum decomposition. As in the case with Hanlon's type A result discussed in Section 1, we call this direct sum decomposition a type B Hodge decomposition.

Theorem 21. Let $C_{r}^{(j)}\left(\Delta_{G}\right)=\tilde{\rho}_{r}^{(j)} C_{r}\left(\Delta_{G}\right)$. The chain complex for $\Delta_{G}$ decomposes as

$$
\left(C_{*}\left(\Delta_{G}\right), \partial_{*}\right)=\bigoplus_{j \geqslant 0}\left(C_{*}^{(j)}\left(\Delta_{G}\right), \partial_{*}\right),
$$

thus

$$
H_{r}\left(\Delta_{G}\right)=\bigoplus_{j \geqslant 0} H_{r}^{(j)}\left(\Delta_{G}\right)
$$

Proof. We will push all of the hard work for the proof to Lemma 23 and Lemma 24. The proof follows easily from from these combined with (3) and (4).

Remark 22. To prove our structural result regarding the $\tilde{\rho}_{n}^{(j)}$, s, it is easier to first work with the elements $\tilde{l}_{n}^{(j)}$ and $\tilde{\lambda}_{n}^{(j)}$ and then apply (5) to convert, as these lemmas and their proofs illustrate. As with the type A Eulerian idempotents discussed in Section 1, the type B Eulerian idempotents can be used to produce a Hodge-type decomposition for commutative hyperoctahedral algebra homology [1]. The chain complex defining this homology is quite similar to the chain complex for the signed graph coloring complex, thus the proofs for Lemmas 23 and 24 are similar to the proof of [1, Proposition 5.1] due to Bergeron and Bergeron. For the sake of completeness, and because [1] contains a few confusing typographical errors, we include reasonably detailed proofs of these results.

Lemma 23. Let $G$ be a signed graph with vertex set $[n]$, with coloring complex $\Delta_{G}$ having chain complex $\left(C_{*}\left(\Delta_{G}\right), \partial_{*}\right)$. For each $r$ such that $0 \leqslant r \leqslant n-2$ and for each $j$ such that $1 \leqslant j \leqslant r+1$,

$$
\partial_{r} \tilde{l}_{r+1}^{(j)}=\left(\tilde{l}_{r}^{(j)}+\tilde{l}_{r}^{(j-1)}\right) \partial_{r}
$$


Proof. Setting

$$
L_{r+1}^{j}:=\left\{\pi \in B_{r+1} \mid \operatorname{des}(\pi)=j\right\},
$$

the strategy is to consider

$$
d_{i} \tilde{l}_{r+1}^{(j)}=d_{i}(-1)^{j-1} \sum_{\pi \in L_{r+1}^{j}} \operatorname{sgn}(\pi) \pi=(-1)^{j-1} \sum_{\pi \in L_{r+1}^{j}} \operatorname{sgn}(\pi) d_{i} \pi
$$

for each $i=1, \ldots, r+1$. For some of the elements $d_{i} \pi \in d_{i} L_{r+1}^{j}$, we will produce $\sigma \in L_{r+1}^{j}$ such that $d_{i} \operatorname{sgn}(\pi) \pi=-d_{i} \operatorname{sgn}(\sigma) \sigma$, and thus these terms will cancel pairwise in (6). For all other elements, we will bijectively map each element in $d_{i} L_{r+1}^{j}$ to an element appearing as a summand of

$$
\begin{aligned}
\left(\tilde{l}_{r}^{(j)}+\tilde{l}_{r}^{(j-1)}\right) \partial_{r} & =\sum_{i=1}^{r+1}(-1)^{i-1}\left((-1)^{j-1} \sum_{\sigma \in L_{r}^{j}} \operatorname{sgn}(\sigma) \sigma+(-1)^{j-2} \sum_{\sigma \in L_{r}^{j-1}} \operatorname{sgn}(\sigma) \sigma\right) d_{i} \\
& =\sum_{i=1}^{r+1}(-1)^{i-1}\left((-1)^{j-1} \sum_{\sigma \in L_{r}^{j}} \operatorname{sgn}(\sigma) \sigma d_{i}+(-1)^{j-2} \sum_{\sigma \in L_{r}^{j-1}} \operatorname{sgn}(\sigma) \sigma d_{i}\right),
\end{aligned}
$$

showing that $d_{i} \pi$ corresponds to a term (with correct sign) $\sigma d_{s}$ in a bijection

$$
\bigcup_{i=1}^{r+1} d_{i} L_{r+1}^{j} \backslash\{\text { pairwise canceling elements }\} \longleftrightarrow\left(\bigcup_{i=1}^{r+1} L_{r}^{j} d_{i}\right) \bigcup\left(\bigcup_{i=1}^{r+1} L_{r}^{j-1} d_{i}\right) .
$$

Doing so will yield our desired equality. As this becomes a lengthy exercise of case-by-case analysis, we will completely prove some of the cases and provide only the setup for the rest.

Case: $i=r+1$.

Let $I_{r+1}$ denote the element of $B_{r+1}$ that sends $r+1$ to $-(r+1)$ and fixes all other elements; note that $\ell\left(I_{r+1}\right)$ is odd, as

$$
I_{r+1}=(r, r+1) \cdots(2,3)(1,2) s_{0}(1,2)(2,3) \cdots(r, r+1),
$$

where $s_{0}$ is the generator of $B_{r+1}$ sending 1 to -1 and fixing all other elements. Next observe that $d_{r+1} I_{r+1}=d_{r+1}$, because when the $(r+1)$-st and $(r+2)$-nd blocks of an ordered partition are merged, all the elements of the $(r+1)$-st block appear in the merged block with all possible signs.

Let $\pi \in L_{r+1}^{j}$ and let $\sigma=I_{r+1} \pi$; it follows from the length of $I_{r+1}$ being odd that $\operatorname{sgn}(\pi)=-\operatorname{sgn}(\sigma)$, by [1, Proposition 1.4.2 (ii)]. Note that $\sigma$ and $\pi$ differ only in that the $\pm(r+1)$ appearing in $\pi$ is negated, but does not change position. If $\pi_{r+1} \neq \pm(r+1)$, then $\sigma=I_{r+1} \pi$ has the same number of descents as $\pi$, since if $r+1$ is in position $j$, then $\pi$ is forced to have an ascent in position $j-1$ and a descent in position $j$, while if $-(r+1)$ is in position $j$, then $\pi$ is forced to have a descent in position $j-1$ and an ascent 
in position $j$. Hence $d_{r+1} \operatorname{sgn}(\sigma) \sigma=d_{r+1} I_{r+1}(-\operatorname{sgn}(\pi)) \pi=-d_{r+1} \operatorname{sgn}(\pi) \pi$, and the pair of terms $d_{r+1} \operatorname{sgn}(\sigma) \sigma$ and $d_{r+1} \operatorname{sgn}(\pi) \pi$ cancel each other in (6).

Suppose now that $\pi_{r+1}=r+1$. Setting $\sigma=\pi_{1} \pi_{2} \cdots \pi_{r}$, it is straightforward to verify that

- $\sigma \in B_{r}$,

- $d_{r+1} \pi=\sigma d_{r+1}$,

- $\operatorname{des}(\sigma)=\operatorname{des}(\pi)$, and

- $\operatorname{sgn}(\pi)=\operatorname{sgn}(\sigma)$.

On the other hand, if $\pi_{r+1}=-(r+1)$, then setting $\sigma=\pi_{1} \pi_{2} \cdots \pi_{r}$ it is again straightforward to verify that

- $\sigma \in B_{r}$,

- $d_{r+1} \pi=\sigma d_{r+1}$,

- $\operatorname{des}(\sigma)=\operatorname{des}(\pi)-1$, and

- $\operatorname{sgn}(\sigma)=-\operatorname{sgn}(\pi)$.

Mapping $d_{r+1} \pi$ to $\sigma d_{r+1}$ in our correspondence yields a bijection (with correct signs) pairing an element $d_{r+1} \pi \in d_{r+1} L_{r+1}^{j}$ satisfying $\pi_{r+1}= \pm(r+1)$ with an element of $L_{r}^{j} d_{r+1} \cup L_{r}^{j-1} d_{r+1}$, leading to the equality

$$
d_{r+1} \tilde{l}_{r+1}^{(j)}=\left(\tilde{l}_{r}^{(j)}+\tilde{l}_{r}^{(j-1)}\right) d_{r+1} .
$$

Case: $1 \leqslant i \leqslant r$.

For each $i$ and each $\pi \in L_{r+1}^{j}$, the relative position of $\pm i$ and $\pm(i+1)$ in the window for $\pi$ determines how $\pi$ is handled. There are five situations that can occur:

- $\pi^{-1}(i+1)=\pi^{-1}(i)+1>0$, implying that $\pi=[\cdots i(i+1) \cdots]$

- $\pi^{-1}(i+1)=\pi^{-1}(i)+1<0$, implying that $\pi=[\cdots-(i+1)-i \cdots]$

- $\pi^{-1}(i+1)=\pi^{-1}(i)-1>0$, implying that $\pi=[\cdots(i+1) i \cdots]$

- $\pi^{-1}(i+1)=\pi^{-1}(i)-1<0$, implying that $\pi=[\cdots-i-(i+1) \cdots]$

- $\pi^{-1}(i+1) \neq \pi^{-1}(i) \pm 1$, containing all remaining cases.

We sketch below how to assign to each $d_{i} \pi$ a unique $\sigma d_{s}$ in each of these cases, and provide at the end a proof that these assignments are bijective as claimed in (7).

Subcase: Suppose $\pi^{-1}(i+1)=\pi^{-1}(i)+1>0$, implying that $\pi=[\cdots i(i+1) \cdots]$.

Define $s$ as the index such that $\pi_{s}=i$. We want to associate to $\pi$ a unique $\sigma \in L_{r}^{j}$ with the following properties: 
- $\operatorname{des}(\sigma)=\operatorname{des}(\pi)$

- $\operatorname{sgn}(\sigma)=(-1)^{i-s} \operatorname{sgn}(\pi)$, and

- $d_{i} \pi=\sigma d_{s}$.

We claim that these properties are satisfied by $\sigma=\sigma_{1} \ldots \sigma_{s} \sigma_{s+2} \ldots \sigma_{r+1}$ where

- $\sigma_{m}=\pi_{m}$ if $\left|\pi_{m}\right|<i+1$

- $\left|\sigma_{m}\right|=\left|\pi_{m}\right|-1$ if $\left|\pi_{m}\right|>i+1$, and

- the sign pattern for $\sigma$ is the same as that for $\pi$, i.e. if $\pi_{j}<0$, then $\sigma_{j}$ is also negative.

To prove that $\operatorname{des}(\sigma)=\operatorname{des}(\pi)$, note that all pairwise inequality relationships are preserved between $\pi$ and $\sigma$; thus, the only possible position of an additional descent in $\pi$ that does not occur in $\sigma$ is between $\pi_{s}=i$ and $\pi_{s+1}=i+1$, where no descent occurs.

To show that $\operatorname{sgn}(\sigma)=(-1)^{i-s} \operatorname{sgn}(\pi)$, observe that there exist $i-s$ adjacent transpositions $t_{1}, \ldots, t_{i-s}$ in $B_{r+1}$ such that $\pi t_{1} \cdots t_{i-s}$ has $i+1$ appearing in window position $i+1$; we do so by exchanging adjacent entries in the window for $\pi$ repeatedly to bring $i+1$ from position $s+1$ to position $i+1$. Then, $\sigma$ is obtained from $\pi t_{1} \cdots t_{i-s}$ by deleting position $i+1$ and lowering the label for all window elements greater than $i+1$. Thus, both $\sigma$ and $\pi t_{1} \cdots t_{i-s}$ can be expressed as a product of the same number of adjacent transpositions and, for each negative element appearing in the window for $\pi$, an odd number of hyperoctahedral group Coxeter generators. Thus, the length of these two elements have the same parity, and our result follows.

Finally, to show $d_{i} \pi=\sigma d_{s}$ it suffices to show that the $m$-th block in the image of $\left(P_{1}|\cdots| P_{r+1} \mid P_{r+2}\right)$ is the same under $d_{i} \pi$ and $\sigma d_{s}$. First, suppose that $\left|\pi_{m}\right|=k<i$. Then $d_{i} \pi$ will map $P_{m}$ to the $k$-th block location in the image if $\pi_{m}>0$, or to $P_{-m}$ in the $k$-th block location in the image if $\pi_{m}<0$. If $m<s$, then $P_{m}$ will still be the $m$-th block in the image after $d_{s}$ is applied. Since $k<i, \sigma_{m}=\pi_{m}$, so $\sigma$ will map $P_{m}$ to the $k$-th block location in the image if $\pi_{m}>0$, or to $P_{-m}$ in the $k$-th block location in the image if $\pi_{m}<0$. If $m>s+1$, then $P_{m}$ will be in the $(m-1)$-st location after $d_{s}$ is applied. Notice though that by the definition of $\sigma$, this implies that $\sigma_{m}$ is in the $(m-1)$-st position of $\sigma$. Since $k<i, \sigma_{m}=\pi_{m}$, and thus $\sigma$ will map $P_{m}$ to the $k$-th block location in the image if $\pi_{m}>0$, or to $P_{-m}$ in the $k$-th block location in the image if $\pi_{m}<0$.

Now suppose that $\left|\pi_{m}\right|=k>i+1$. Then $d_{i} \pi$ will map $P_{m}$ to the $(k-1)$-st block in the image if $\pi_{m}>0$ or to $P_{-m}$ in the $(k-1)$-st block in the image if $\pi_{m}<0$. If $m<s$, then $P_{m}$ will still be the $m$-th block in the image after $d_{s}$ is applied. Since $k>i+1$, $\left|\sigma_{m}\right|=\left|\pi_{m}\right|-1$, so $\sigma$ will map $P_{m}$ to the $(k-1)$-st block location in the image if $\pi_{m}>0$, or to $P_{-m}$ in the $(k-1)$-st block location in the image if $\pi_{m}<0$. If $m>s+1$, then $P_{m}$ will be in the $(m-1)$-st location after $d_{s}$ is applied. Notice though that by the definition of $\sigma$, this implies that $\sigma_{m}$ is in the $(m-1)$-st position of $\sigma$. Since $k>i+1,\left|\sigma_{m}\right|=\left|\pi_{m}\right|-1$, and thus $\sigma$ will map $P_{m}$ to the $(k-1)$-st block location in the image if $\pi_{m}>0$, or to $P_{-m}$ in the $(k-1)$-st block location in the image if $\pi_{m}<0$. 
Now suppose that $m=s$. Then $d_{i} \pi$ will map $P_{s}$ to $P_{s} \cup P_{s+1}$ in the $i$-th location. $d_{s}$ will map $P_{s}$ to $P_{s} \cup P_{s+1}$, and since $P_{s} \cup P_{s+1}$ is in the $s$-th block, $\sigma d_{s}$ also maps $P_{s}$ to $P_{s} \cup P_{s+1}$ in the $i$-th block.

Subcase: Suppose $\pi^{-1}(i+1)=\pi^{-1}(i)+1<0$, implying that $\pi=[\cdots-(i+1)-i \cdots]$.

Let $s$ be defined as the index such that $\pi_{s}=-i-1$. It suffices to show that $\sigma$ defined as follows satisfies $\operatorname{des}(\sigma)=\operatorname{des}(\pi), \operatorname{sgn}(\sigma)=(-1)^{i-s} \operatorname{sgn}(\pi)$, and $d_{i} \pi=\sigma d_{s}$. Let $\sigma=\sigma_{1} \ldots \sigma_{s} \sigma_{s+2} \ldots \sigma_{r+1}$ where

- $\sigma_{m}=\pi_{m}$ if $\left|\pi_{m}\right|<i$

- $\left|\sigma_{m}\right|=\left|\pi_{m}\right|-1$ if $\left|\pi_{m}\right|>i$, and

- the sign pattern for $\sigma$ is the same as that for $\pi$, i.e. if $\pi_{j}<0$, then $\sigma_{j}$ is also negative.

Thus, $\sigma d_{s}$ is the element in $L_{r}^{j} d_{s}$ uniquely paired with $d_{i} \pi$. This argument is similar to the previous subcase.

Subcase: Suppose $\pi^{-1}(i+1)=\pi^{-1}(i)-1>0$, implying that $\pi=[\cdots(i+1) i \cdots]$.

Let $s$ be defined as the index such that $\pi_{s}=i+1$. It suffices to show that $\sigma$ defined as follows satisfies, $\operatorname{des}(\sigma)=\operatorname{des}(\pi)-1, \operatorname{sgn}(\sigma)=(-1)^{i-s+1} \operatorname{sgn}(\pi)$, and $d_{i} \pi=\sigma d_{s}$. Let $\sigma=\sigma_{1} \ldots \sigma_{s-1} \sigma_{s+1} \ldots \sigma_{r+1}$ where

- $\sigma_{m}=\pi_{m}$ if $\left|\pi_{m}\right|<i+1$,

- $\left|\sigma_{m}\right|=\left|\pi_{m}\right|-1$ if $\left|\pi_{m}\right|>i+1$, and

- the sign pattern for $\sigma$ is the same as that for $\pi$, i.e. if $\pi_{j}<0$, then $\sigma_{j}$ is also negative.

Thus, $\sigma d_{s}$ is the element of $L_{r}^{j-1} d_{s}$ uniquely paired with $d_{i} \pi$. This argument is similar to the previous subcase. Note that the property $\operatorname{sgn}(\sigma)=(-1)^{i-s+1} \operatorname{sgn}(\pi)$ is necessary because while $\pi \in L_{r+1}^{j}$, we have that $\sigma \in L_{r}^{j-1}$ and thus in $\tilde{l}_{r}^{(j-1)}$ we have that $\operatorname{sgn}(\sigma) \sigma$ is multiplied by $(-1)^{j-1}$ rather than $(-1)^{j}$.

Subcase: Suppose $\pi^{-1}(i+1)=\pi^{-1}(i)-1<0$, implying that $\pi=[\cdots-i-(i+1) \cdots]$.

Let $s$ be the index such that $\pi_{s}=-i$. It suffices to show that $\sigma$ defined as follows satisfies $\operatorname{des}(\sigma)=\operatorname{des}(\pi)-1, \operatorname{sgn}(\sigma)=(-1)^{i-s+1} \operatorname{sgn}(\pi)$, and $d_{i} \pi=\sigma d_{s}$. Let $\sigma=$ $\sigma_{1} \ldots \sigma_{s-1} \sigma_{s+1} \ldots \sigma_{r+1}$ where

- $\sigma_{m}=\pi_{m}$ if $\left|\pi_{m}\right|<i$,

- $\left|\sigma_{m}\right|=\left|\pi_{m}\right|-1$ if $\left|\pi_{m}\right|>i$, and

- the sign pattern for $\sigma$ is the same as that for $\pi$, i.e. if $\pi_{j}<0$, then $\sigma_{j}$ is also negative. 
Thus, $\sigma d_{s}$ is the element of $L_{r}^{j-1} d_{s}$ uniquely paired with $d_{i} \pi$. This argument is similar to the previous subcase, and the same comment as in the previous subcase about $\operatorname{sgn}(\sigma)=$ $(-1)^{i-s+1} \operatorname{sgn}(\pi)$ applies.

Subcase: Suppose $\pi^{-1}(i+1) \neq \pi^{-1}(i) \pm 1$.

Setting $\sigma=(i, i+1) \pi$, one can show that $\operatorname{sgn}(\pi)=-\operatorname{sgn}(\sigma), \operatorname{des}(\pi)=\operatorname{des}(\sigma)$, and that $d_{i} \pi=d_{i} \sigma$. Thus, the terms corresponding to $d_{i} \pi$ and $d_{i} \sigma$ cancel in (6).

Unique bijection: To see that our correspondences above are bijective, consider an element $\sigma d_{s} \in L_{r}^{j} d_{s}$, where $\sigma=\sigma_{1} \cdots \sigma_{s-1} \sigma_{s} \sigma_{s+1} \cdots \sigma_{r}$. Then $\sigma d_{s}$ is obtainable from some $d_{j} \pi$ via our above process if $\sigma$ was obtained by deleting the $s$-th or $(s+1)$-st element from $\pi$, i.e.

Case A: $\sigma=[\sigma_{1} \cdots \sigma_{s} \underbrace{}_{\begin{array}{c}\pi_{s+1} \\ \text { dropped } \\ \text { here }\end{array}} \sigma_{s+1} \cdots \sigma_{r}]$ or Case B: $\sigma=[\sigma_{1} \cdots \sigma_{s-1} \underbrace{}_{\begin{array}{c}\pi_{s} \\ \text { dropped } \\ \text { here }\end{array}} \sigma_{s} \cdots \sigma_{r}]$.

Considering our claimed bijective map described above, in both Case A and Case B it is the element $\sigma_{s}$ that determined which element of $\pi$ was dropped. Suppose that $\sigma_{s}= \pm i$. For each of Case A and Case B, a fixed parity for $\sigma_{s}$ yields a unique $\pi, j$ such that $d_{j} \pi$ maps to $\sigma d_{s}$ under our map, and hence our claimed bijection (7) is established.

\section{Lemma 24.}

$$
\partial_{r} \tilde{\lambda}_{r+1}^{(j)}=\tilde{\lambda}_{r}^{(j)} \partial_{r} \quad \text { and } \quad \partial_{r} \tilde{\rho}_{r+1}^{(j)}=\tilde{\rho}_{r}^{(j)} \partial_{r}
$$

Proof. Since

$$
\tilde{\lambda}_{r+1}^{(j)}=\sum_{i=0}^{j}(-1)^{i}\left(\begin{array}{c}
r+1+i \\
i
\end{array}\right) \tilde{l}_{r+1}^{(j-i)}
$$

it follows from Lemma 23 that

$$
\begin{aligned}
& \partial_{r} \tilde{\lambda}_{r+1}^{(j)}=\partial_{r} \sum_{i=0}^{j}(-1)^{i}\left(\begin{array}{c}
r+1+i \\
i
\end{array}\right) \tilde{l}_{r+1}^{(j-i)} \\
& =\sum_{i=0}^{j}(-1)^{i}\left(\begin{array}{c}
r+1+i \\
i
\end{array}\right)\left(\tilde{l}_{r}^{(j-i)}+\tilde{l}_{r}^{(j-i-1)}\right) \partial_{r} \\
& =\left[(-1)^{0}\left(\begin{array}{c}
r+1 \\
0
\end{array}\right)\left(\tilde{l}_{r}^{(j)}+\tilde{l}_{r}^{(j-1)}\right)+(-1)^{1}\left(\begin{array}{c}
r+2 \\
1
\end{array}\right)\left(\tilde{l}_{r}^{(j-1)}+\tilde{l}_{r}^{(j-2)}\right)+\cdots\right. \\
& \left.+(-1)^{j-1}\left(\begin{array}{c}
r+1+j-1 \\
j-1
\end{array}\right)\left(\tilde{l}_{r}^{(1)}+\tilde{l}_{r}^{(0)}\right)+(-1)^{j}\left(\begin{array}{c}
r+1+j \\
j
\end{array}\right) \tilde{l}_{r}^{(0)}\right] \partial_{r} \\
& =\left[(-1)^{0}\left(\begin{array}{c}
r+1 \\
0
\end{array}\right) \tilde{l}_{r}^{(j)}+\left((-1)^{0}\left(\begin{array}{c}
r+1 \\
0
\end{array}\right)+(-1)^{1}\left(\begin{array}{c}
r+2 \\
1
\end{array}\right)\right) \tilde{l}_{r}^{(j-1)}+\cdots\right. \\
& \left.+\left((-1)^{j-1}\left(\begin{array}{c}
r+1+j-1 \\
j-1
\end{array}\right)+(-1)^{j}\left(\begin{array}{c}
r+1 \\
j
\end{array}\right)\right) \tilde{l}_{r}^{0}\right] \partial_{r}
\end{aligned}
$$




$$
\begin{aligned}
& =\sum_{i=0}^{j}(-1)^{i}\left(\begin{array}{c}
r+1 \\
i
\end{array}\right) \tilde{l}_{r}^{(j-i)} \partial_{r} \\
& =\tilde{\lambda}_{r}^{(j)} \partial_{r},
\end{aligned}
$$

which establishes the first claim.

For the second claim, note that due to the relation

$$
\tilde{\lambda}_{r+1}^{(j)}=(-1)^{j} \tilde{\rho}_{r+1}(2 j+1)
$$

it follows that the $\tilde{\lambda}_{r+1}^{(j)}$ 's and the $\tilde{\rho}_{r+1}^{(j)}$ 's are related by an invertible Vandermonde matrix. Changing basis in this manner from the first to the second set of elements establishes the second claim.

\section{Chromatic polynomial coefficients and Hodge de- compositions}

In this section we establish that the coefficients of $\chi_{G}(\lambda)$ encode (up to sign) the dimensions of the Hodge components for $H_{n-2}\left(\Delta_{G}\right)$. First we must establish that Hodge decompositions are preserved by switching.

Lemma 25. If two signed graphs $G$ and $H$ are switching equivalent, then there is a chain complex isomorphism between $C_{*}\left(\Delta_{G}\right)$ and $C_{*}\left(\Delta_{H}\right)$ respecting the Hodge decompositions.

Proof. Suppose that $H$ is obtained from $G$ by switching at vertex $i$. It is straightforward to check that the map $f_{i}: C_{*}\left(\Delta_{G}\right) \rightarrow C_{*}\left(\Delta_{H}\right)$ obtained by exchanging $i$ and $-i$ in every face of $\Delta_{G}$ is a chain complex isomorphism; one way to see this is to recognize that $\Delta_{G}=B_{G} \cap \partial[-1,1]^{n}$ is taken to $\Delta_{H}=B_{H} \cap \partial[-1,1]^{n}$ by the map $x_{i} \rightarrow-x_{i}$, and this induces the map $f_{i}$ at the level of chain complexes. Using the combinatorial description of coloring complexes given in Definition 14 and the action of $B_{r+1}$ on $C_{r}$ defined for any coloring complex, it is immediate that for any $\pi \in B_{r+1}$ we have

$$
\pi \circ f_{i}=f_{i} \circ \pi
$$

hence

$$
\tilde{\rho}_{r+1}^{(j)} \circ f_{i}=f_{i} \circ \tilde{\rho}_{r+1}^{(j)} \cdot
$$

Our lemma follows by combining this with the fact that $f_{i}$ is a chain complex isomorphism.

Theorem 26. Let $G$ be a signed graph on $[n]$ with at least one edge or half-edge. Writing

$$
\chi_{G}(\lambda)=\lambda^{n}+\sum_{j=0}^{n-1}(-1)^{n-j} c_{j} \lambda^{j},
$$


we have $\operatorname{dim} H_{n-2}^{(j)}\left(\Delta_{G}\right)=c_{j}$. Equivalently,

$$
(-1)^{n}\left[\chi_{G}(-\lambda)-(-\lambda)^{n}\right]=\sum_{j=0}^{n} c_{j} \lambda^{j}=\sum_{j=0}^{n} \operatorname{dim} H_{n-2}^{(j)}\left(\Delta_{G}\right) \lambda^{j} .
$$

Proof. We go by induction on $n$, similar to the proof given by Hanlon [8, Theorem 4.1].

Base Case: Suppose first that $E$ consists of a single half-edge; without loss of generality, we can consider this half-edge to be $\{n\}$. Then $\Delta_{G} \cong S^{n-2}$, so $\operatorname{dim} H_{n-2}\left(\Delta_{G}\right)=1$. Let $\gamma=(1|2| \cdots|n-1|-1-2 \cdots-n n)$; let

$$
\Gamma:=\left[\frac{1}{2^{n-1}(n-1) !} \sum_{\sigma \in B_{n-1}} \operatorname{sgn}(\sigma) \sigma\right] \gamma .
$$

Claim: $\partial \Gamma=0$. Considering the application of each $d_{i}$ independently, we obtain

$$
\begin{aligned}
& \sum_{i=1}^{n-1}(-1)^{i-1} d_{i} \cdot \Gamma=\sum_{i=1}^{n-1} \frac{1}{2^{n-1}(n-1) !} \sum_{\sigma \in B_{n-1}}(-1)^{i-1} d_{i} \operatorname{sgn}(\sigma) \sigma \gamma \\
= & \sum_{i=1}^{n-1} \frac{1}{2^{n-1}(n-1) !} \sum_{\sigma \in B_{n-1}}(-1)^{i-1} d_{i} \operatorname{sgn}(\sigma)\left(\gamma_{\sigma^{-1}(1)}|\cdots| \gamma_{\sigma^{-1}(n-1)} \mid \gamma_{n}^{\prime}\right) \\
= & \sum_{i=1}^{n-1} \frac{1}{2^{n-1}(n-1) !}(-1)^{i-1} \sum_{\sigma \in B_{n-1}} \operatorname{sgn}(\sigma)\left(\gamma_{\sigma^{-1}(1)}|\cdots| \gamma_{\sigma^{-1}(i)} \cup \gamma_{\sigma^{-1}(i+1)}|\cdots| \gamma_{\sigma^{-1}(n-1)} \mid \gamma_{n}^{\prime}\right) .
\end{aligned}
$$

For $i \neq n-1$, on the terms of the sum $\sum_{\sigma \in B_{n-1}} \operatorname{sgn}(\sigma) d_{i} \sigma$, consider the involution $\sigma \rightarrow(i, i+1) \sigma$. This yields a sign-reversing involution on the summands in the final displayed line above.

On the terms of the corresponding sum for $d_{n-1}$, consider the involution $\sigma \rightarrow I_{n-1} \sigma$, where

$$
I_{n-1}=(n-2, n-1) \cdots(2,3)(1,2) s_{0}(1,2)(2,3) \cdots(n-2, n-1) .
$$

This yields another sign-reversing involution on the summands in the final displayed line above, hence

$$
\partial \Gamma=0
$$

Since $\Gamma=\tilde{\rho}_{n-1}^{(n-1)}$, it follows that

$$
\operatorname{dim} H_{n-2}^{(n-1)}\left(\Delta_{G}\right)=1
$$

Since $\chi_{G}(\lambda)=(\lambda-1) \lambda^{n-1}=\lambda^{n}-\lambda^{n-1}$, our base case holds.

In the case where $E$ consists of only an edge, we can without loss of generality consider the edge to be $\{n-1, n\}$. If we set $\gamma=(1|2| \cdots|\{n, n-1\}|-1-2 \cdots-n)$, then the same analysis as given above holds, establishing this base case as well.

Induction: Let $G$ be a signed graph with $n \geqslant 2$ edges, and assume by way of induction that Theorem 26 holds for any signed graph with fewer than $n$ edges. Let $e$ be an edge 
of $G$; without loss of generality, we may assume $e$ is either a half-edge or a positive edge, since by Proposition 7 and Lemma 25 we may switch a negative $e$ to obtain a new signed graph with the same chromatic polynomial and Hodge structure. Let $E$ be the graph with vertex set $V(G)$ and edge set $\{e\}$. Let $C_{r}(E)$ denote the space spanned by the chains $P=\left(P_{1}|\cdots| P_{r+2}\right)$ that contain $e$. Let $D_{r}$ denote the space spanned by the chains $P=\left(P_{1}|\cdots| P_{r+2}\right)$ in $\Delta_{G}$ that do not contain $e$. It follows that

$$
C_{r}(G)=D_{r} \oplus C_{r}(E)
$$

Notice that the action of $B_{r+2}$ commutes with the isomorphism, and thus,

$$
C_{r}^{(j)}(G)=D_{r}^{(j)} \oplus C_{r}^{(j)}(E)
$$

Considering $D_{r}$ next, we claim that

$$
D_{r} \simeq C_{r}(G \backslash e) /\left(C_{r}(G \backslash e) \cap C_{r}(E)\right) .
$$

To prove this, observe that $C_{r}(G \backslash e)$ has as a spanning set the set of chains $P=\left(P_{1}|\cdots| P_{r+2}\right)$ where at least one of the $P_{i}$ contains an edge of the graph $G \backslash e$. This spanning set consists of chains $P$ that contain an edge of the graph $G \backslash e$ as well as the edge $e$, and it consists of chains $P$ that contain an edge of the graph $G \backslash e$ but do not also contain the edge $e$. The set of all chains $P$ that contain an edge of $G \backslash e$ but not the edge $e$ form a spanning set for $C_{r}(G \backslash e) /\left(C_{r}(G \backslash e) \cap C_{r}(E)\right)$. Notice that this set is also a spanning set for $D_{r}$, and the isomorphism then follows. Since the action $B_{r+2}$ commutes with this isomorphism, we have

$$
D_{r}^{(j)} \simeq C_{r}^{(j)}(G \backslash e) /\left(C_{r}^{(j)}(G \backslash e) \cap C_{r}^{(j)}(E)\right) .
$$

We finally claim that

$$
C_{r}^{(j)}(G \backslash e) \cap C_{r}^{(j)}(E) \simeq C_{r}^{(j)}(G / e)
$$

Suppose first that $e=\{a, b\}$ is a positive edge. Consider the map sending

$$
P=\left(P_{1}\left|P_{2}\right| \cdots\left|P_{r+1}\right| P_{r+2}\right) \in C_{r}(G \backslash e) \cap C_{r}(E)
$$

to the chain $Q=\left(Q_{1}|\cdots| Q_{r+2}\right) \in C_{r}(G / e)$ obtained by replacing the pair $\{a, b\}$ by the symbol $a=b$ representing the contracted vertex in $G / e$. It is straightforward that this map gives a bijection inducing our desired isomorphism between $C_{r}(G \backslash e) \cap C_{r}(E)$ and $C_{r}(G / e)$, because the pair of symbols $\{a, b\}$ in any basis chain $P \in C_{r}(G \backslash e) \cap C_{r}(E)$ is simply replaced by the contracted vertex symbol $a=b$. Note that surjectivity follows since any chain $Q \in C_{r}(G / e)$ will contain an edge of $G / e$ in some block, which will by definition correspond to an edge in $G \backslash e$, and hence a preimage under our map may be found. It is clear that this map is invariant under the hyperoctahedral group action, as $a$ and $b$ always are moved as part of the same block in both settings.

Next, suppose that $e=\{j\}$ is a half-edge, and observe that $C_{r}(E)$ is spanned by chains with $j,-j$ in $P_{r+2}$. Consider the map which takes a chain $\left(P_{1}|\cdots| P_{r+2}\right) \in C_{r}(G \backslash e) \cap C_{r}(E)$ 
and deletes the $j,-j$ in $P_{r+2}$ to obtain a chain $\left(P_{1}|\cdots| P_{r+2}\{j,-j\}\right) \in C_{r}(G / e)$. We claim that this is a bijection inducing our desired isomorphism. To prove this, note that for any chain $\left(Q_{1}|\cdots| Q_{r+2}\right) \in C_{r}(G / e)$ we can add $\{j,-j\}$ to $Q_{r+2}$ and obtain a new chain. This is actually a chain in the spanning set for $C_{r}(G \backslash e) \cap C_{r}(E)$; that it is in $C_{r}(E)$ is clear. To see that it is in $C_{r}(G \backslash e)$, we consider two possible cases. First, if a block $Q_{k}$ contains an edge in $G$ not incident to $j$, in which case this is also an edge in $G \backslash e$, our chain is a spanning element of $C_{r}(G \backslash e)$ and we are done. Second, if no block $Q_{k}$ contains an edge in $G / e$, then for some $i$ where $i, j$ is an edge of $G$, we must have that $i,-i$ is in $Q_{r+2}$. Then when we add in $j,-j$ to $Q_{r+2}$, we have all of $i, j,-i,-j$ in $Q_{r+2}$. This implies that $\left(Q_{1}|\cdots| Q_{r+2} \cup\{j,-j\}\right)$ can be obtained by merging the last two blocks in $\left(Q_{1}|\cdots| Q_{r+1}|\{i, j\}|\left(Q_{r+2} \backslash\{i\}\right) \cup\{-j\}\right)$, and this longer chain corresponds to a spanning element of $C_{r+1}(G \backslash e)$. Thus, $\left(Q_{1}|\cdots| Q_{r+2} \cup\{i,-i\}\right)$ must also be in the spanning set $C_{r}(G \backslash e)$. It is immediate that these maps are invariant under the hyperoctahedral group action, as the final block containing $j,-j$ is always fixed by the group.

From (8), (9), and (10), it follows that

$$
\operatorname{dim}\left(C_{r}^{(j)}(G)\right)=\operatorname{dim}\left(C_{r}^{(j)}(G \backslash e)\right)-\operatorname{dim}\left(C_{r}^{(j)}(G / e)\right)+\operatorname{dim}\left(C_{r}^{(j)}(E)\right) .
$$

Using the fact that the reduced homology $H_{*}\left(\Delta_{G}\right)$ is only nonvanishing in top dimension, along with the Euler-Poincare identity and our inductive hypothesis, we conclude that

$$
\begin{aligned}
& \sum_{j} \operatorname{dim}\left(H_{n-2}^{(j)}(G)\right) \lambda^{j} \\
= & \sum_{j, r}(-1)^{(n-2)-r} \operatorname{dim}\left(H_{r}^{(j)}(G)\right) \lambda^{j} \\
= & \sum_{j, r}(-1)^{n-2-r} \operatorname{dim}\left(C_{r}^{(j)}(G)\right) \lambda^{j} \\
= & \sum_{j, r}(-1)^{n-2-r}\left(\operatorname{dim}\left(C_{r}^{(j)}(G \backslash e)\right)-\operatorname{dim}\left(C_{r}^{(j)}(G / e)\right)+\operatorname{dim}\left(C_{r}^{(j)}(E)\right) \lambda^{j}\right. \\
= & \sum_{j} \operatorname{dim}\left(H_{n-2}^{(j)}(G \backslash e)\right) \lambda^{j}-(-1) \sum_{j} \operatorname{dim}\left(H_{n-1}^{(j)}(G / e)\right) \lambda^{j}+\sum_{j} \operatorname{dim}\left(H_{n-2}^{(j)}(E)\right) \lambda^{j} \\
= & (-1)^{n}\left[\left(\chi_{G \backslash e}(-\lambda)-(-\lambda)^{n}\right)-\left(\chi_{G / e}(-\lambda)-(-\lambda)^{n-1}\right)+\left(\chi_{E}(-\lambda)-(-\lambda)^{n}\right)\right] \\
= & (-1)^{n}\left[\chi_{G \backslash e}(-\lambda)-\chi_{G / e}(-\lambda)-(-\lambda)^{n}\right] \\
= & (-1)^{n}\left(\chi_{G}(-\lambda)-(-\lambda)^{n}\right) .
\end{aligned}
$$

Remark 27. At the end of his paper regarding type A Hodge decompositions and ordinary chromatic polynomials, Hanlon [8] states that it would be interesting to find ways to use properties of chromatic polynomials to prove algebraic results about Hodge decompositions of coloring complexes, and vice versa. It is reasonable to ask for similar uses of Theorem 26 in the context of signed graphs. The existence of these results in both type A 
and type B are evidence that such connections might be waiting to be uncovered, though at present they remain elusive.

\section{Acknowledgements}

The authors thank the anonymous referee for their thoughtful suggestions.

\section{References}

[1] F. Bergeron and N. Bergeron, Orthogonal idempotents in the descent algebra of $B_{n}$ and applications, J. Pure Appl. Algebra, 79 (1992), 109-129.

[2] A. Björner and F. Brenti, Combinatorics of Coxeter groups. Graduate Texts in Mathematics, 231. Springer, New York, 2005.

[3] F. Breuer, A. Dall, and M. Kubitzke, Hypergraph coloring complexes, Discrete Math. 312 (2012), no. 16, 2407-2420.

[4] P. Diaconis and J. Fulman, Foulkes characters, Eulerian idempotents, and an amazing matrix, J. Algebraic Combin. 36 (2012), no. 3, 425-440.

[5] A. Garsia, Combinatorics of the free Lie algebra and the symmetric group, Analysis, et cetera, 309-382, Academic Press, Boston, MA, 1990.

[6] M. Gerstenhaber and S.D. Schack, A Hodge-type decomposition for commutative algebra cohomology, J. Pure Appl. Algebra, 48 (1987), 229-247

[7] P. Hanlon, The action of $S_{n}$ on the components of the Hodge decomposition of Hochschild homology, Michigan Math. J., 37 (1990) 1, 105-124.

[8] P. Hanlon, A Hodge decomposition interpretation for the coefficients of the chromatic polynomial, Proc. Amer. Math. Soc., 136 (2008), 3741-3749.

[9] P. Hanlon and P. Hersh, A Hodge decomposition for the complex of injective words, Pacific J. Math. 214 (2004), no. 1, 109-125.

[10] P. Hersh and E. Swartz, Coloring complexes and arrangements, J. Algebraic Combin. 27 (2008), no. 2, 205-214.

[11] J. Herzog, V. Reiner, and V. Welker, The Koszul property in affine semigroup rings, Pacific J. Math. 186 (1998), no. 1, 39-65.

[12] A. Hultman, Link complexes of subspace arrangements, European J. Combin., 28 (2007), 781-790.

[13] J. Jonsson, The topology of the coloring complex, J. Algebraic Combin., 21 (2005), 311-329.

[14] J-L Loday, Partition eulérienne et opérations en homologie cyclique, C. R. Acad. Sci. Paris Sér. I Math., Comptes Rendus des Séances de l'Académie des Sciences. Série I. Mathématique, 307 (1988), no. 7, 283-286. 
[15] J. Long and S. Rundell, The Hodge structure of the coloring complex of a hypergraph, Discrete Math. 311 (2011), no. 20, 2164-2173.

[16] C. Reutenauer, Theorem of Poincaré-Birkhoff-Witt, logarithm and symmetric group representations of degrees equal to Stirling numbers, Combinatoire énumérative (Montreal, Que., 1985/Quebec, Que., 1985), 267-284, Lecture Notes in Math., 1234, Springer, Berlin, 1986.

[17] S. Rundell, The coloring complex and cyclic coloring complex of a complete $k$-uniform hypergraph, J. Combin. Theory Ser. A, 119 (2012), 1095-1109.

[18] E. Steingrímsson, The coloring ideal and coloring complex of a graph, J. Algebraic Combin., 14 (2001), 73-84.

[19] T. Zaslavsky, Signed graphs, Discrete Appl. Math., Vol 4, 1982, no 1, 47-74.

[20] T. Zaslavsky, Signed graph coloring, Discrete Math., Vol 39, 1982, no 2, 215-228. 